\title{
Construction of exact solutions by spatial traslations in inhomogeneous Nonlinear Schrödinger equations.
}

\author{
Juan J. García-Ripoll, Víctor M. Pérez-García and Vadym Vekslerchik $\uplus$ \\ Departamento de Matemáticas, E. T. S. I. Industriales, Universidad de Castilla-La Mancha, \\ Avenida de Camilo José Cela, 3, 13071 Ciudad Real, Spain.
}

(November 3, 2018)

\begin{abstract}
In this paper we study a general nonlinear Schrödinger equation with a time dependent harmonic potential. Despite the lack of traslational invariance we find a symmetry trasformation which, up from any solution, produces infinitely many others which are centered on classical trajectories. The results presented here imply that, not only the center of mass of the wave-packet satisfies the Ehrenfest theorem and is decoupled from the dynamics of the wave-packet, but also the shape of the solution is independent of the behaviour of the center of the wave. Our findings have implications on the dynamics of Bose-Einstein condensates in magnetic traps.
\end{abstract}

PACS number(s): 05.45.Yv, 03.75.Fi, 42.65.Tg

\section{INTRODUCTION}

One of the most fruitful concepts of Physics is that of symmetries. From high-energy physics to condensed matter, symmetries play a central role on our understanding of the world.

As what concerns classical field theories, a symmetry is a transformation which preserves the form of the equations. In this case the symmetry can help us in many different ways. First of all, me may build solutions which have the same symmetry as the equation. Let us take the two-dimensional spatially homogeneous nonlinear Schrödinger (NLS) equation

$$
i \partial_{t} \psi(\mathbf{r}, t)=\left[-\frac{1}{2} \triangle+|\psi|^{2}\right] \psi(\mathbf{r}, t) .
$$

This equation is invariant under spatial rotations, and therefore we are able to search solutions with the given symmetry, $\psi=\psi\left(\sqrt{x^{2}+y^{2}}\right)(x+i y)^{n}$.

Second and most important, Noether's theorem ensures us that once we have found a certain symmetry in our model, it is possible to construct certain quantities, often with physical relevance, which will be conserved during the evolution. For instance, the invariance of Eq. (1) under time translations, spatial translations and rotations, give us seven conserved quantities, which are the energy

$$
E[\psi]=\int|\nabla \psi|^{2}+\frac{1}{4}|\psi|^{4},
$$

the linear moment of the center of mass

$$
\mathbf{P}_{c}=\frac{d}{d t}\langle\mathbf{r}\rangle=\langle-i \nabla\rangle \equiv \int-i \bar{\psi} \nabla \psi,
$$

and the angular momentum of the wavepacket

$$
\mathbf{L}=\langle-i \mathbf{r} \times \nabla\rangle .
$$

Finally, the existence of symmetries and their associated conservation laws helps us in the study of other properties. In particular, the invariance of Eq. (11) under Galilean transformations allows us to rewrite our equations on an inertial frame of reference where the center of mass is still. This means that the dynamics of the center of mass does not affect at all the dynamics of other properties of the wavepacket.

In this paper we study a generalization of Eq. (11) which lacks traslational invariance. Nevertheless we will show a Galilean-like symmetry, which allows us to construct, from any solution, a continuum of other ones which follow different classical trajectories. We will show that this symmetry implies a decoupling of the dynamics of the center of mass with respect to all other properties of the wavepacket. We will also point out some very relevant applications of our findings to the dynamics of Bose-Einstein condensates and of several optical systems.

\section{THE MODEL}

In this work we will consider the following family of nonlinear Schrödinger (NLS) equations with a general nonlinear term $G(|\psi|)$

$$
i \partial_{t} \psi(\mathbf{r}, t)=\left[-\frac{1}{2} \triangle+V(\mathbf{r}, t)+G(|\psi|)\right] \psi(\mathbf{r}, t) .
$$

We will restrict our interest to the case of a quadratic potential $V(\mathbf{r}, t)$, i.e.

$$
V(\mathbf{r}, t)=\frac{1}{2}(\mathbf{r}, A(t) \mathbf{r}), \quad A_{i j}=\omega_{i}(t) \delta_{i j} .
$$

Eq. (5) with potential (6) is an accurate model of many physical phenomena. In particular it describes the dynamics of a Bose-Einstein condensate in the mean field approximation [1], the propagation of optical beams in graded index fibers [2] and the propagation of solitary waves in fiber trasmission lines with in-line phase modulators [3]. 
The nonlinear term, $G$, may adopt many different forms depending on the particular application of Eq. (6). The most classical cases are the so called power nonlinearities $G\left(|\psi|^{2}\right)= \pm|\psi|^{p}$ which arise in mean-field models with different spatial dimensionalities. In Nonlinear Optics we also find many versions of the so called saturable nonlinearities, e.g. $G\left(|\psi|^{2}\right)= \pm|\psi|^{2} /\left(1+\beta|\psi|^{2}\right)$ as well as their Taylor aproximations for small $u, G\left(|\psi|^{2}\right)=$ $\pm|\psi|^{2}-\alpha|\psi|^{4}$. But the nonlinearity need not be local, and in applications to Bose-Einstein condensation one finds nonlocal expansions of the atom-atom interaction $G\left(|\psi|^{2}\right)=\int K\left(\mathbf{r}-\mathbf{r}^{\prime}\right)\left|\psi\left(\mathbf{r}^{\prime}\right)\right|^{2} d \mathbf{r}^{\prime}$, where the kernel is either radially symmetric $K\left(\left|\mathbf{r}-\mathbf{r}^{\prime}\right|\right)$ [1, 1 or adopts more complex dependencies in the case of dipole-dipole interactions [5]. These are only a few examples of the many forms the nonlinear term may have.

The description of the dynamics involved in a NLS equation is of great interest for applications. However, except for the very specific one dimensional case with $G= \pm|\psi|^{2}, A=0$, in which the equation may be integrated by means of the Inverse Scattering method, nothing can be said about the structure of the solutions. There are other tools such as the moment method which give us information about the evolution of relevant integral quantities characterizing the solution [2]. In some cases, these methods are connected to the conformal invariance of some classes of nonlinear Schrödinger equations [6] but have several limitations: (i) They cannot be used to build explicit solutions of the equations and (ii) they work exactly only on specific cases. To derive a procedure which is valid for more general nonlinear problems as the ones we consider here one must use some nontrivial approximations [7].

In this paper we will be able to exploit the behavior of Eq. (5) with harmonic potential (6) under spatial traslations to provide explicit information on a whole class of time dependent problems as will be shown below.

\section{BUILDING NEW SOLUTIONS OF THE NLS BY SPATIAL TRASLATIONS.}

\section{A. General case}

Let us consider a solution $\psi(\mathbf{r}, t)$ of Eqs. (5)-(6) satisfying $\psi(\mathbf{r}, t=0)=\xi(\mathbf{r})$. Our main result is that given any solution, $\psi(\mathbf{r}, t)$, there exists a continuum of other solutions which are of the form

$$
\psi_{\mathbf{R}}(\mathbf{r}, t)=\psi(\mathbf{r}-\mathbf{R}(t), t) e^{i \theta(\mathbf{r}, t)},
$$

being $\mathbf{R}(t)$ and $\theta(\mathbf{r}, t)$ appropriate functions to be determined later.

To check this point we proceed by inserting the ansatz $\psi_{\mathbf{R}}(\mathbf{r}, t)$ given by Eq. (7) into Eq. (5). Using the fact that $\psi(\mathbf{r}, t)$ is a solution of Eq. (5), we are able to cancel several terms on both sides of the equation. If we impose that the new function $\psi_{\mathbf{R}}$ be also a solution of Eq. (5), we reach a solvability condition which is made up of all the remaining terms

$$
\begin{aligned}
& i\left(\nabla \theta-\frac{d \mathbf{R}}{d t}, \nabla \psi\right)= \\
& \frac{1}{2}\left[\partial_{t} \theta-i \triangle \theta+(\nabla \theta)^{2}+(2 \mathbf{r}-\mathbf{R}, A(t) \mathbf{R})\right] \psi .
\end{aligned}
$$

This is a set of partial differential equation for the unknown function $\theta(\mathbf{r}, t)$. Fortunately, it is possible to construct solutions by choosing a linear phase,

$$
\theta(\mathbf{r}, t)=\left(\mathbf{r}, \frac{d \mathbf{R}}{d t}\right)+f(t)
$$

together with a trajectory $\mathbf{R}(t)$ determined by equations of Newton type

$$
\frac{d^{2} \mathbf{R}}{d t^{2}}+A(t) \mathbf{R}=0
$$

By applying the hydrodynamic interpretation of the NLS equation [9], the precise for of Eq. (9) leads to a divergenceless velocity field, $\mathbf{v}=\nabla \theta=\mathbf{R}$, which is responsible for the global displacement of the solution.

Finally, we need a global contribution to the phase, $f(t)$, which is determined uniquely from

$$
\frac{d f}{d t}=\left(\frac{d \mathbf{R}}{d t}, \frac{d \mathbf{R}}{d t}\right)-(\mathbf{R}, A(t) \mathbf{R}) .
$$

This contribution can be calculated for each trajectory,

$$
f(t)=\int_{0}^{t}\left[\left(\frac{d \mathbf{R}}{d t}, \frac{d \mathbf{R}}{d t}\right)-(\mathbf{R}, A(t) \mathbf{R})\right] d t .
$$

Therefore, what we get from Eqs. (7), (9), (10) and (12) is a new solution of Eq. (5) which is displaced from the initial one. It is remarkable that these explicit timedependent solutions are obtained by spatial traslations in a system which is not spatially homogeneous and the dynamics is defined by simple, linear ordinary differential equations. This behavior is exclusive of the harmonic oscillator type potential given by Eq. (6) but it is not restricted to any specific form of the nonlinear term or any dimensionality of the system.

\section{B. Evolution of stationary states}

A relevant type of solutions of Eq. (5) are the so called solitary waves or stationary solutions, which are of the form

$$
\psi(\mathbf{r}, t)=\phi_{\mu}(\mathbf{r}) e^{i \mu t}
$$

The existence and number of these solutions depends on the properties of the nonlinear term. In this paper we will assume that the nonlinear term is such that these solutions exist, which is in fact the case for most choices 
of $G$ of physical interest [4, 8]. Then we may build from (13) new solutions of the type

$$
\phi_{(\mathbf{R}, \mu)}(\mathbf{r}, t)=\phi_{\mu}(\mathbf{r}-\mathbf{R}(t)) e^{i[\mu t+\theta(\mathbf{r}, t)]} .
$$

In this case the whole of the wavepacket moves following a classical orbit, while preserving the shape! This interesting prediction can be confirmed both experimentally and numerically.

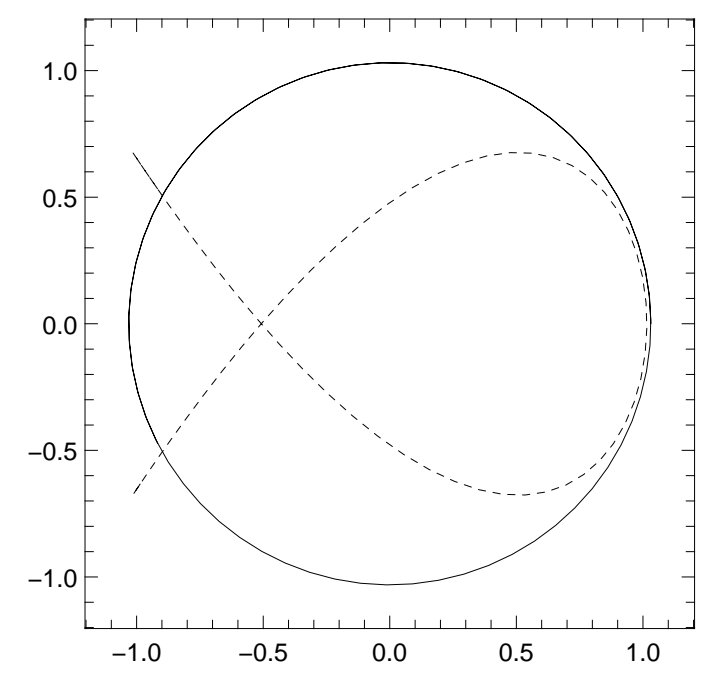

FIG. 1. Trajectories of a solution which is initially stationary, and is suddenly displaced and imparted an initial velocity. We plot the closed trajectories of the center of mass in the symmetric confinement (solid line, $\omega_{x}=\omega_{y}=1$, $\mathbf{R}(0)=(1,0), \dot{\mathbf{R}}(0)=(0,1))$ and in the asymmetric trap (dashed line, $\omega_{y}=1.2, \omega_{x}=1, \mathbf{R}(0)=(1,0), \mathbf{R}(0)=(0,1)$ ). The trajectories have been obtained integrating Eq. (5) numerically.

In Fig. 1] we show the evolution of two of such wavepackets, first in the symmetric trap (solid line) and in the asymmetric trap (dashed line). Such solutions were obtained by solving Eq. (5) using a split-step method on a Fourier basis with $128 \times 128$ modes. As our analysis predicts, the shape of the wavefunction is preserved up to the numerical precision of the computer.

\section{Addition of rotational terms.}

The proof presented in Sec. III A is also valid when the matrix $A(t)$ is non diagonal. An specific case of physical interest arises in Bose-Einstein condensation when the trap which confines the atoms rotates. In that case it is customary to study the system on the frame of reference which moves with the trap, at angular speed $\Omega(t)$. On these coordinates the NLS equation reads

$$
i \partial_{t} \psi=\left[-\frac{1}{2} \triangle+V(\mathbf{r})+G(|\psi|)+\Omega L_{z}\right] \psi
$$

where $L_{z}$ is the Hermitian operator which represents the projection of the angular momentum along the rotation axis and is given by

$$
L_{z} \psi=-i(\mathbf{r}, J \nabla \psi) .
$$

The antisymmetric matrix, $J$, is the generator of the rotations around the $z$ axis

$$
J=\left(\begin{array}{ccc}
0 & 1 & 0 \\
-1 & 0 & 0 \\
0 & 0 & 0
\end{array}\right)
$$
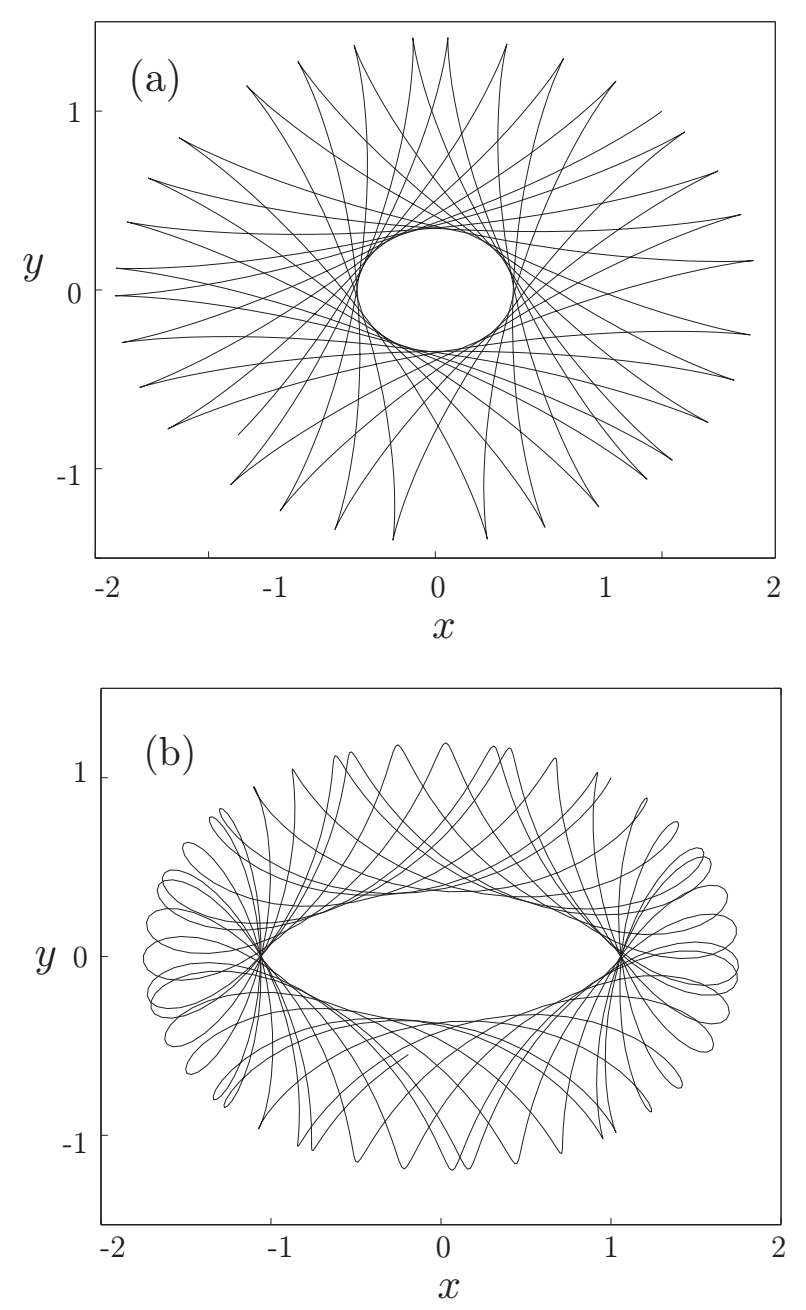

FIG. 2. Trajectories of the center of a solution placed initially at $x(0)=1, y(0)=1$, with $\dot{R}(0)=0$ in two different situations: (a) $\omega_{x}=\omega_{y}=1, \Omega=1 / 2$, (b) $\omega_{x}=1, \omega_{y}=3 / 2, \Omega=3 / 2$.

By repeating the same calculations one arrives to a classical equation for the wavepacket center, $\mathbf{R}(t)$, with an additional term due to the centrifugal force

$$
\frac{d^{2} \mathbf{R}}{d t^{2}}+\Omega J \frac{d \mathbf{R}}{d t}+A \mathbf{R}=0 .
$$


These equations form a linear system whose solutions are easy to obtain. Specifically, for a two-dimensional oscillator and constant frequencies, $\omega_{x}, \omega_{y}$ and $\Omega$, the solutions are quasiperiodic with characteristic frequencies given by

$$
\begin{aligned}
& \omega_{1}^{2}= \mid \sqrt{\left(\omega_{x}^{2}+\omega_{y}^{2}\right)^{2}+\Omega^{2}\left(\Omega^{2}+2\left(\omega_{x}^{2}+\omega_{y}^{2}\right)\right)} \\
&+\omega_{y}^{2}-\Omega^{2}-\omega_{x}^{2} \mid \\
& \omega_{2}^{2}=\mid \Omega^{2}+\omega_{x}^{2}-\omega_{y}^{2} \\
&+\sqrt{\left(\omega_{x}^{2}+\omega_{y}^{2}\right)^{2}+\Omega^{2}\left(\Omega^{2}+2\left(\omega_{x}^{2}+\omega_{y}^{2}\right)\right)} \mid .
\end{aligned}
$$

Typical solutions are plotted in Fig. 2, where the trajectory of the wavepacket has been integrated numerically up from Eq. (18). It is important to stress the stability of these solutions: even in the case of overcritical rotation $\left(\Omega>\omega_{x, y}\right)$, when the centrifugal force exceeds the restoring force of the harmonic potential, the motion is made of bounded oscillations.

Incidentally, there is a formal equivalence between a NLS equation with a rotating trap (15) with $\omega_{x}=\omega_{y}=\Omega$ and a Ginzburg-Landau equation

$$
i \partial_{t} \psi=\frac{1}{2}(-i \nabla+\mathbf{A})^{2} \psi+|\psi|^{2} \psi
$$

with uniform magnetic field, $\mathbf{A}^{t}=\Omega(y,-x, 0)$. In this model it is particularly intuitive that the wavepacket should rotate around the origin, due to the action of the uniform magnetic field, just as the above discussed symmetry (16) reveals.

\section{DECOUPLING THE DYNAMICS OF THE CENTER OF MASS}

Up to now we have shown that given a solution $\psi(\mathbf{r}, t)$, we can build many others, $\psi_{\mathbf{R}}(\mathbf{r}, t)$, by spatial traslations of the initial data. The process can be reversed, so that given a wavepacket $\phi \equiv \psi_{\mathbf{R}}(\mathbf{r}, t)$ which is a solution of Eq. (5) we can extract the dynamics of the center of mass, $\mathbf{R}(\mathbf{t})$, and the internal dynamics of the wavepacket, $\psi(\mathbf{r}, \mathbf{t})$.

The practical process is as follows. Let $\phi(\mathbf{r}, t)$ be any solution of the NLS equation with a harmonic potential (5). The center of mass position is defined as

$$
\mathbf{R}_{c}(t)=\langle\mathbf{r}\rangle \equiv \int \mathbf{r}|\phi(\mathbf{r}, t)|^{2} d^{n} \mathbf{r}
$$

The dynamics of the center of mass, and of its associated momentum (3), is given by Ehrenfest's equations. Using the notation from Quantum Mechanics, the expected value of an operator $A$ evolves according to

$$
\frac{d}{d t} A=\langle i[H(\psi), A]\rangle
$$

where $H(\psi)$ is a nonlinear operator given by

$$
H=-\frac{1}{2} \triangle+V(\mathbf{r}, t)+G(|\psi|) .
$$

Applying Eq. (23) to $\mathbf{r}$ and to $(-i \nabla)$, we obtain the following coupled ordinary differential equations

$$
\begin{aligned}
\frac{d}{d t} \mathbf{R}_{c} & =\langle-i \nabla\rangle=\mathbf{P}_{c} \\
\frac{d}{d t} \mathbf{P}_{c} & =\langle-\nabla V\rangle=-A \mathbf{R}_{c} .
\end{aligned}
$$

With some manipulations it is easy to rewrite this system as a second order differential equation

$$
\frac{d^{2} \mathbf{R}_{c}}{d t^{2}}+A \mathbf{R}_{c}=0
$$

with initial conditions

$$
\begin{aligned}
\mathbf{R}_{c}(0) & =\int \mathbf{r}|\xi(\mathbf{r})|^{2} d^{n} r \\
\left.\frac{d \mathbf{R}_{c}}{d t}\right|_{t=0} & =-i \int \bar{\xi} \nabla \xi d^{n} r
\end{aligned}
$$

where $\xi(\mathbf{r})=\phi(\mathbf{r}, 0)$ is the initial data of Eq. (5).

This means that the center of mass already satisfies the equations for a valid displacement in our symmetry transformation (7). Hence we can define a second wavefunction, $\psi(\mathbf{r}, t)$, which moves with the center of mass, and which is the solution of Eq. (5) with initial data

$$
\psi(\mathbf{r}, 0)=\xi\left(\mathbf{r}+\mathbf{R}_{c}\right) \exp \left(-i \mathbf{r}, \frac{d \mathbf{R}_{c}}{d t}\right) .
$$

This second wavefunction, $\psi(\mathbf{r}, t)$, is located on the center of mass

$$
\int \mathbf{r}|\psi(\mathbf{r}, t)|^{2} d^{n} r=0
$$

and it is the one that carries the dynamics of all observables — widths, angular momentum, circulation, etccompletely free from the influence of the center of mass.

Summing up, what all these transformations tell us is that if we displace the initial data, or impart some speed to its center, we obtain the same solution, $\psi(\mathbf{r}, t)$, centered on different trajectories.

This result has been obtained with the help of the Erhenfest theorem, which states that the center of mass should satisfy an equation of Newton type, and which was already known 10]. However, the result summarized in Eq. (30) is much stronger since it states that the wavepacket is not affected by the dynamics of its centrum, as this dynamics can be integrated out of the equations.

\section{APPLICATION TO THE DYNAMICS OF THE CENTER OF MASS IN BOSE-EINSTEIN CONDENSATES.}

Ever since the first works with dilute Bose-Einstein condensates, there has been an amazingly precise agreement between theory and experiments. From the studies 
of normal modes, to the nucleation of vortices, it is usual to obtain a good quantitative matching between the predictions (let it be collective frequencies or critical speeds) and the actual measurements.

This is most intriguing in the case of experiments which involve a mechanical perturbation of the condensate. We first focus on the study of the collective excitations of a condensate. Such experiments consist of a periodic modulation of the confinement of the condensate, and the subsequent study of the oscillations of the wavepacket's widths. These manipulations have been shown to not only modulate the widths, but to induce an exact, and extremely strong resonance of the center of mass [10,11. Nevertheless, both in the experimental results and in some rough models, the widths and the center of mass seem to be decoupled, thus allowing us to precisely characterize the normal modes of the condensate. That observed behavior is easy to understand in the framework of the dynamics of displaced solutions described here.

Another important application of Eq. (10) is the study of the center of mass of the condensate in the regime of overcritical rotation, $\Omega>\min \left\{\omega_{x}, \omega_{y}\right\}$. In this regime the rotating condensate, which is ruled by Eq. (15), suffers a centrifugal force which is stronger than the restoring force due to the harmonic potential. It is clear that in this regime the condensate should be, and in fact it is found to be [12], untrapped.

However, the analysis of the eigenvalues of Eqs. (18), which are given by (19) proves that the equilibrium point at $x=y=0$ is a center and thus dynamically stable. Therefore, the only source of instability for the condensate under overcritical rotations can be due to deformations of the cloud.

This result is a bit more general than the one in [13], where it is proposed the existence of some configurations for the condensate, which correspond to centered and elliptically deformed clouds that survive to the action of the centrifugal motion. These configurations are stable under dipolar perturbations (displacements of the cloud) and under quadrupolar excitations (certain type of deformations). It remains an open problem to show whether such states exist which are dynamically stable under any deformation.

As a side result which can be verified in experiments, a perturbed condensate in a rotating trap suffers bounded oscillations around the origin with two different frequencies, $\omega_{1}$ and $\omega_{2}$. These frequencies bear a nontrivial dependency with respect to the angular speed of the trap (19), which can be used to better calibrate experiments. Finally we must remark that the existence of two different oscillation frequencies for the center of mass, $\omega_{1}$ and $\omega_{2}$, even in the symmetric trap $\left(\omega_{x}=\omega_{y}\right)$ represents a splitting of the dipolar mode, which is intuitively similar to the splitting of the quadrupolar mode due to the presence of a vortex.

\section{CONCLUSIONS AND DISCUSSION.}

In this paper we have built new solutions by simple time dependent traslations in a system without traslational symmetry. It is remarkable, and probably a special feature of the harmonic potential that this procedure works. Specially striking is the case of traslation of stationary solutions whose center moves harmonically whithout any distortion on the shape of the solution itself (only a simple phase appears).

In relation with the previous finding, we have shown that the dynamics of the center of mass is decoupled from the dynamics of all other properties of the wavepacket. This result stands on other works 10,11. However, the contribution of this paper is different and stronger, since we show that motion of the center of mass can never influence any other properties of the wavepacket, let it be a Bose-Einstein condensate or, in a similar row, a solitary wave made of light. For all these systems the evolution will be essentially the same, no matter the initial position and initial velocity of the atomic cloud or solitary wave.

Our calculations are valid for any type of nonlinearity which is symmetric under translations, and which depends only on the density, $|\psi|$. This includes the cubic nonlinearity for Bose-Einstein condensates, $G=|\psi|^{2}$, and most reasonable nonlocal terms $\llbracket$. This, and the fact that our calculations do not depend on the dimensionality of the system, extends the validity of this work to condensates with dipolar interactions, charged condensates, light in Kerr media and light in saturable media.

The decoupling of the motion of the center of mass has also practical consequences. The invariance of the wavepacket dynamics up to displacements and impulses on the initial data, explains why it is actually possible to measure the frequencies of the normal modes of a condensate, even when the center of mass of the condensate is known to be exponentially influenced by the changes on the trapping potential [10,11]. This invariance also benefits experiments with rotating condensates, as we have shown above, and a simple analysis reveals an unexpected splitting of the dipolar mode of a condensate.

The situation is different when other type of potentials are considered such as stationary pinning potentials or any non-harmonic trapping potential, such as some polynomial candidates, $V(x) \propto x^{4}$, which are being considered in the context of all-optical condensation in very elongated traps. The presence of such potentials breaks our calculations, as the dynamics of the center of mass couples to that of the widths by means of these external agents. We wonder if this will imply some new and puzzling dynamics in future experiments.

\section{ACKNOWLEDGMENTS}

This work has been supported by grant BFM20000521. V. Vekslerchik is supported by Ministerio de Edu- 
cación, Cultura y Deporte under grant SB99-AH777133.

+ On leave from Institute for Radiophysics and Electronics, Kharkov 61085, Ukraine.

[1] F. Dalfovo, S. Giorgini, L. Pitaevski, S. Stringari, Rev. Mod. Phys. 71, 463-512 (1999).

[2] J. J. García-Ripoll, V. M. Pérez-García, and P. Torres, Phys. Rev. Lett. 30, 1715 (1999); V. M. Pérez-García, P. Torres, J. J. García-Ripoll, H. Michinel, J. Opt. B: Quantum Semiclass. Opt. 2, 353 (2000).

[3] N. Smith, F. M. Knox, N. J. Doran, K. J. Blow, and I. Bennion, Electron. Lett. 32, 55 (1995); I. Gabitov, E. Shapiro, and S. Turitsyn, Phys. Rev. E 55, 3624 (1997); S. Kumar and A. Hasegawa, Opt. Lett. 22, 372 (1997); S. Turitsyn, Phys. Rev. E 56, R3784 (1997).

[4] V. M. Pérez-García, V. V. Konotop, and J. J. GarcíaRipoll, Phys. Rev. E. 62, 4300 (2000).

[5] D. O'Dell, S. Giovanazzi, G. Kurizky, V. M. Akulin, Phys. Rev. Lett. 84, 5687 (2000); L. Santos, G. V. Schlyapnikov, P. Zoller, M. Lewenstein Phys. Rev. Lett. 85, 1791 (2000).

[6] P. K. Ghosh, cond-mat/0102488.

[7] V. M. Pérez-García, J. J. García-Ripoll, P. Torres, SIAM J. Appl. Math. (in preparation).

[8] M. Kunze, T. Küpper, V.K. Mezentsev, E.G. Shapiro and S. Turitsyn, Physica D 128, 273 (1999).

[9] J. J. García-Ripoll, and V. M. Pérez-García, Phys. Rev. A 64, 013602 (2001).

[10] J. J. García-Ripoll, and V. M. Pérez-García, Phys. Rev. A 59, 2220 (1999).

[11] J. J. García-Ripoll, V. M. Pérez-García, and P. Torres, Phys. Rev. Lett. 30, 1715 (1999).

[12] K. W. Madison, F. Chevy, W. Wohlleben, and J. Dalibard, Phys. Rev. Lett. 84, 806 (2000).

[13] A. Recati, F. Zambelli, and S. Stringari, Phys. Rev. Lett. 86, 377 (2001). 